\title{
Errores en las recetas médicas y en la preparación de estas en farmacia de pacientes ambulatorios. El caso del Hospital de Nueva Imperial
}

\author{
CAROLINA ALVARADO A. ${ }^{1, a}$, XIMENA OSSA G. ${ }^{2, \mathrm{~b}}$, LUIS BUSTOS M.2,c
}

\section{Errors in prescriptions and their preparation at the outpatient pharmacy of a regional hospital}

Background: Adverse effects of medications are an important cause of morbidity and hospital admissions. Errors in prescription or preparation of medications by pharmacy personnel are a factor that may influence these occurrence of the adverse effects Aim: To assess the frequency and type of errors in prescriptions and in their preparation at the pharmacy unit of a regional public hospital. Material and Methods: Prescriptions received by ambulatory patients and those being discharged from the hospital, were reviewed using a 12-item checklist. The preparation of such prescriptions at the pharmacy unit was also reviewed using a seven item checklist. Results: Seventy two percent of prescriptions had at least one error. The most common mistake was the impossibility of determining the concentration of the prescribed drug. Prescriptions for patients being discharged from the hospital had the higher number of errors. When a prescription had more than two drugs, the risk of error increased 2.4 times. Twenty four percent of prescription preparations had at least one error. The most common mistake was the labeling of drugs with incomplete medical indications. When a preparation included more than three drugs, the risk of preparation error increased 1.8 times. Conclusions: Prescription and preparation of medication delivered to patients had frequent errors. The most important risk factor for errors was the number of drugs prescribed.

(Rev Med Chile 2017; 145: 33-40)

Key words: Community Pharmacy Services; Inappropriate Prescribing; Medication Errors; Pharmacology; Pharmacy.

$\mathrm{D}$ esde la publicación en el año 2000 del informe "To err is human: Building a safer health system", del Instituto de Medicina de Estados Unidos de Norteamérica (EE.UU.), la seguridad del paciente dentro de los hospitales ha mejorado, debido a una serie de medidas legislativas y reglamentarias impulsadas por las autoridades, dada la importante repercusión asistencial y económica que tienen los daños prevenibles derivados de errores durante la asistencia sanitaria ${ }^{1,2}$.

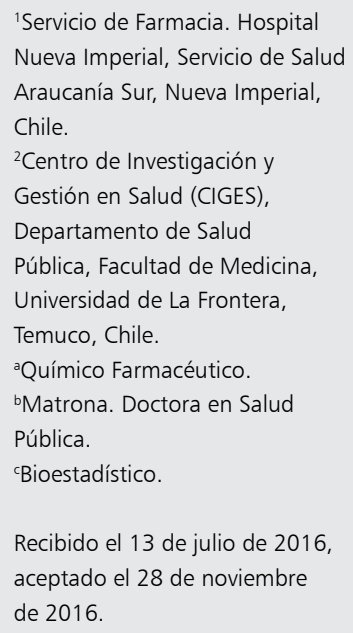

Correspondencia a:

Carolina Alvarado

Teléfono: +56985497135

caalvarado@gmail.com
Entre los incidentes derivados de la asistencia clínica, lo más prevalentes son los errores asociados al uso de medicamentos, que pueden tener lugar en cualquier etapa del circuito de utilización ${ }^{3}$. Los errores de medicación son definidos como: "cualquier incidente prevenible que puede causar daño al paciente o dar lugar a una utilización inapropiada de los medicamentos, cuando estos están bajo control de profesionales sanitarios o

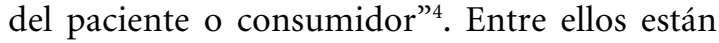


los errores en las recetas médicas que provienen de fallas en la escritura de la receta, que da lugar a una instrucción errónea acerca de una o más de las "características normales" de una receta (identidad de quien la recibe, identidad de la droga, formulación, dosis, vía, tiempo, frecuencia y duración de la administración) $)^{5}$. Se agregan, además, los errores de dispensación, que se definen como la discrepancia entre lo que indica una receta médica y el medicamento dispensado por la farmacia al paciente, o que distribuye a la sala, sobre la base de esta receta, incluyendo la dispensación de un medicamento con inferior calidad farmacéutica o de información ${ }^{6}$. Si este error es detectado antes que los medicamentos lleguen al paciente, se denomina "dispensing near miss"; para el presente estudio se entenderá este último como un error en la preparación de las recetas, pesquisado antes de entregar los medicamentos al paciente ${ }^{7}$.

Aunque las tasas de errores en la dispensación son generalmente bajas, las mejoras en los sistemas de distribución de farmacia siguen siendo importantes, porque estas dispensan volúmenes tan altos de medicamentos que incluso una baja tasa de error puede traducirse en un gran número de errores ${ }^{8}$. En pacientes ambulatorios, la incidencia de acontecimientos prevenibles por medicamentos es de 5,4\%. Algunos autores han determinado, además, que la tasa de error oscila entre 3,5 y $11.4 \%$ de las dosis administradas, y que un paciente hospitalizado presenta 0,9 errores de medicación diarios ${ }^{4,9,10}$. Cuarenta y dos por ciento de los eventos adversos potencialmente mortales o graves se deben a errores en la prescripción, en donde la tasa de error oscila entre 6,3 y $14,7 \%$ de errores en las recetas manuscritas ${ }^{11-13}$.

Como no existe una estimación de la magnitud y las características de los errores de medicación en el hospital de Nueva Imperial, esta investigación tiene como principal objetivo determinar la prevalencia y tipo de errores, tanto en las recetas, como en su preparación y los factores asociados a estos errores, tales como el número de prescripciones por receta, número de técnicos paramédicos que participan de la preparación, horario de recepción y servicio clínico de procedencia. Para ello se utilizaron pautas de chequeo, previamente diseñadas y validadas, proceso que fue dado a conocer en esta misma Revista en una publicación anterior ${ }^{14}$.

\section{Material y Método}

El presente es un estudio descriptivo de corte transversal realizado en el año 2013 en la Farmacia de Atención Ambulatoria del Hospital de Nueva Imperial, establecimiento de mediana complejidad, perteneciente a la red del Servicio de Salud Araucanía Sur, que recibe pacientes de las comunas de Nueva Imperial, Carahue y Puerto Saavedra. Esta farmacia dispensa recetas de pacientes crónicos atendidos en policlínico de medicina interna, pacientes de morbilidad atendidos en policlínico de otras especialidades (traumatología, pediatría, oftalmología entre otros), de urgencia y también a pacientes que una vez finalizado su proceso de hospitalización son dados de alta con tratamiento farmacológico. Las recetas son preparadas por dos técnicos paramédicos, que van intercalando cada paso del proceso de preparación de recetas (el que prepara no es el mismo que entrega) de manera de realizar un doble control del proceso. El químico farmacéutico supervisa estas actividades, centrándose principalmente en la entrega de los medicamentos.

Las unidades de investigación fueron dos: la receta médica y la preparación de la prescripción. Se consideró una población total de 67.024 recetas, basándose en el promedio anual de recetas dispensadas en farmacia los dos años anteriores al estudio.

El cálculo de muestra para las recetas médicas consideró una prevalencia de errores de $4 \%$, basada en un estudio piloto de 40 recetas elegidas al azar y respaldada por lo publicado por Otero ${ }^{4}$, con un nivel de confianza del $95 \%$, y $0,6 \%$ de precisión, lo que determinó finalmente un $\mathrm{n}=3.862$. La selección de las recetas fue en forma aleatoria, comenzando con un número al azar y, posteriormente, una de cada doce se seleccionaba para ser evaluada, hasta llegar a las 16 diarias necesarias para completar el número de recetas determinado para estudiar en un año. Se determinó este período de estudio (un año) para asegurar la representatividad de la variabilidad estacional que se produce en la dinámica, número y tipos de fármacos dispensados.

El cálculo de muestra para evaluar errores en la preparación de las recetas consideró una prevalencia de errores de $17 \%$, basada en el análisis de un estudio piloto de 156 preparaciones de recetas común nivel de confianza del 95\%, y 
2,6\% de precisión, lo que determinó finalmente un $\mathrm{n}=824$. La selección de las preparaciones para evaluar fue por conveniencia, donde el investigador escogió los días y el momento en cada bloque horario (mañana, mediodía, tarde) para realizar la selección de las preparaciones, de manera de completar 18 evaluaciones semanales. Se definió esta metodología, ya que se trataba de un proceso contante, instantáneo, no probabilístico.

\section{Recolección de datos}

Se utilizó una pauta de chequeo diferente para cada unidad de investigación, las cuales fueron elaboradas y validadas previamente ${ }^{14}$. La pauta para medir errores en las recetas médicas quedó conformada por 12 ítems representando posibles errores en las características de la receta y la pauta para medir errores en la preparación por 7 ítems, que representan posibles errores en las acciones durante la preparación. Se aplicó la pauta de chequeo de lunes a viernes, en forma aleatoria, a 16 recetas del total del día. En relación con la preparación de recetas, se aplicó la pauta inmediatamente después de preparada la receta y antes de entregar al paciente los medicamentos, a tres preparaciones por cada bloque horario, en horario hábil, dos días a la semana.

Se analizaron los errores en las recetas médicas y su preparación, con sus respectivas frecuencias. Se evaluó asociación entre el número de errores en las recetas médicas y el servicio de procedencia y número de prescripciones por receta. Así como también entre el número de errores en la preparación de recetas y el número de prescripciones por receta, número de técnicos paramédicos que las preparaban y horario de recepción. Para el análisis se utilizó pruebas estadísticas de Kruskal-Wallis, $\mathrm{T}$ test para varianzas distintas y un modelo de regresión lineal múltiple, usando software estadístico Stata $12^{\circledR}$.

\section{Resultados}

Se evaluaron 3.872 recetas médicas y 827 preparaciones de recetas, en forma independiente. $\mathrm{La}$ frecuencia de las características estudiadas para cada unidad de análisis se detalla en la Tabla 1.

En cuanto a las recetas médicas, encontramos que $72,1 \%$ de las recetas presentaba al menos un error. Los errores de mayor prevalencia fueron imposibilidad de determinar la concentración del medicamento prescrito y ausencia de identificación del médico prescriptor. El mayor número de errores encontrados en una receta fue de 8 y lo más frecuente fue encontrar un error por receta (Tabla 2).

Las recetas con mayor promedio de errores correspondieron a las provenientes de las altas médicas, observándose diferencias significativas $(p=0,0001)$ en los promedios de errores de los diferentes servicios de procedencia de las recetas. Las recetas con más de 3 fármacos prescritos presentaron un mayor promedio de errores con respecto a aquellas que poseían 2 o menos fármacos por receta $(\mathrm{p}<0,0001)$ (Tabla 3$)$. Cuando se analizó en general la probabilidad de errores, este aumentó en 2,4 veces por cada unidad de fármaco que se agrega (Tabla 4). Si este análisis se realiza por servicio de procedencia, se observó que cuando la receta tenía más de dos fármacos, la probabilidad de error era mayor en las recetas que provenían de las altas médicas ( 3,6 veces) y del policlínico de medicina interna ( 3,5 veces) (Tabla 5 ).

En cuanto a la preparación de recetas, encontramos que $23,9 \%$ presentaba al menos un error. El

\section{Tabla 1. Frecuencia de las características estudiadas}

\begin{tabular}{|lrr|}
\hline Unidad de investigación & \multicolumn{2}{c}{ Frecuencia } \\
& n & $\%$ \\
Preparación de recetas & & \\
Número de fármacos por receta & 546 & 66 \\
- 1-3 & 281 & 33 \\
- 4 y más & & \\
Número de Técnico Paramédicos & & \\
- 1 & 141 & 17 \\
- 2 & 686 & 83 \\
Bloque horario & & \\
- Mañana & 266 & 32 \\
- Mediodía & 289 & 35 \\
- Tarde & 272 & 33 \\
Receta Médica & & \\
Número de fármacos por receta. & & \\
- 1-2 & 2.809 & 73 \\
- 3 y más & 1.063 & 27 \\
Servicio de procedencia de la receta médica & & \\
- Alta médica paciente hospitalizado & 212 & 5 \\
- Policlínico de Medicina Interna & 460 & 12 \\
- Policlínico Especialidades & 773 & 20 \\
- Urgencia & 2.427 & 63 \\
\hline
\end{tabular}


Tabla 2. Distribución de errores en las recetas médicas según tipo y frecuencia

\begin{tabular}{|c|c|c|}
\hline Variable & Frecuencia & $\%$ \\
\hline \multicolumn{3}{|l|}{ Tipo de Error } \\
\hline - No es posible determinar identificación del paciente & 510 & 13,2 \\
\hline - No es posible determinar concentración del medicamento & 1.263 & 32,6 \\
\hline - No es posible determinar la forma farmacéutica del medicamento & 597 & 15,4 \\
\hline - No es posible determinar la vía de administración del medicamento & 631 & 16,3 \\
\hline - No es posible determinar dosis diaria del medicamento & 485 & 12,5 \\
\hline - No es posible determinar duración del tratamiento & 878 & 22,7 \\
\hline - No es posible determinar cantidad total correcta del medicamento & 638 & 16,5 \\
\hline - Prescripción repetida de un medicamento en la misma receta & 13 & 0,3 \\
\hline - Uso de abreviaturas, símbolos o siglas en la prescripción de medicamentos & 789 & 20,4 \\
\hline - Posee enmendaduras en las indicaciones de los medicamentos & 206 & 5,3 \\
\hline - Ausencia de identificación del médico & 952 & 24,6 \\
\hline - Ausencia firma del médico & 9 & 0,2 \\
\hline \multicolumn{3}{|l|}{ Número de errores por receta } \\
\hline$\cdot 0$ & 1.081 & 27,9 \\
\hline - 1 & 1.047 & 27,0 \\
\hline - 2 & 599 & 15,5 \\
\hline - 3 & 473 & 12,2 \\
\hline - 4 & 313 & 8,1 \\
\hline - 5 & 178 & 4,6 \\
\hline - 6 & 110 & 2,8 \\
\hline - 7 & 63 & 1,6 \\
\hline - 8 & 8 & 0,2 \\
\hline
\end{tabular}

Tabla 3. Distribución y promedio de errores en las recetas médicas según servicio de procedencia y número de fármacos

\begin{tabular}{|c|c|c|c|c|c|}
\hline Variables & $\begin{array}{l}\text { Frecuencia } \\
\text { de errores }\end{array}$ & $\%$ & $\begin{array}{l}\text { Promedio } \\
\text { de errores }\end{array}$ & $\begin{array}{l}\text { Desviación } \\
\text { Estándar }\end{array}$ & $\mathbf{p}$ \\
\hline \multicolumn{6}{|l|}{ Servicio de Procedencia Receta Médica } \\
\hline - Alta médica paciente hospitalizado & 212 & 5,5 & 2,5 & 1,89 & $0,0001 *$ \\
\hline - Policlínico de Medicina Interna & 460 & 11,9 & 1,9 & 1,68 & \\
\hline - Policlínicos Especialidades & 773 & 20,0 & 1,5 & 1,97 & \\
\hline - Urgencia & 2.427 & 62,7 & 1,8 & 1,70 & \\
\hline \multicolumn{6}{|l|}{ Número de fármacos } \\
\hline - 1 у 2 & 2.809 & 72,5 & 1,6 & 1,70 & $<0,0001^{* *}$ \\
\hline - 3 y más & 1.063 & 27,5 & 2,3 & 1,85 & \\
\hline
\end{tabular}

*Kruskal-Wallis. ${ }^{* *}$ T test para varianzas distintas.

tipo de error que se presentó con mayor frecuencia fue la rotulación de medicamentos con indicaciones médicas incompletas, seguido por aquellos donde el medicamento se rotuló con indicaciones médicas incorrectas. El mayor número de errores detectados por receta fue de 3 , y lo más frecuente fue un error por preparación (Tabla 6).

El bloque horario de medio día presentó mayor promedio de errores en las preparaciones de recetas con respecto a los otros bloques, pero 
Tabla 4. Riesgo de error según procedencia de recetas médicas con dos o más fármacos. Regresión logística

\begin{tabular}{|lcccc|}
\hline Variable & OR & SD & p & IC (95\%) \\
\hline Alta médica paciente hospitalizado & 3,68 & 0,74 & $<0,0001$ & $2,49-5,45$ \\
\hline Urgencia & 3,32 & 0,29 & $<0,0001$ & $2,79-3,95$ \\
\hline Policlínico de Medicina Interna & 1,74 & 0,24 & $<0,0001$ & $1,33-2,26$ \\
Número de fármaco $=2$ & 2,35 & 0,23 & $<0,0001$ & $1,94-2,85$ \\
\hline
\end{tabular}

*Referencia: Policlínico de especialidades.

Tabla 5. Riesgo de error según procedencia de recetas médicas con dos o más fármacos. Regresión logística

\begin{tabular}{|lccc|}
\hline Variable & OR & SD & p \\
\hline Alta médica paciente hospitalizado & 3,59 & 1,44 & 0,002 \\
\hline Urgencia & 3,04 & 0,51 & $<0,0001$ \\
\hline Policlínico de Medicina Interna & 3,49 & 0,78 & $<0,0001$ \\
Policlínicos Especialidades & 1,3 & 0,22 & 0,116 \\
\hline
\end{tabular}

Tabla 6. Distribución de errores en la preparación de recetas según tipo y frecuencia

\begin{tabular}{|lrr|}
\hline Variable & Frecuencia & $\%$ \\
\hline Tipo de Error & 37 & 4,5 \\
- Medicamentos rotulados con indicaciones médicas incorrectas & 131 & 15,8 \\
- Medicamentos rotulados con indicaciones médicas incompletas & 10 & 1,2 \\
- Medicamento empacado no corresponde al rotulado en la etiqueta & 1 & 0,1 \\
- Medicamento no corresponde a la forma farmacéutica recetada & 12 & 1,5 \\
- No se preparó uno o más medicamentos prescritos en la receta médica & 19 & 2,3 \\
- Cantidad total del medicamento empacado incorrecto & 5 & 0,6 \\
- Hay preparación de medicamentos que no están prescritos en la receta & 629 \\
Número de errores por preparación & 183 & 76,1 \\
- 0 & 13 & 22,1 \\
- 1 & 2 & 1,6 \\
- 2 & 2 & 0,2 \\
\hline
\end{tabular}

esta diferencia no fue significativa $(\mathrm{p}=0,3743)$. El promedio de errores cuando había sólo un técnico paramédico al momento de la preparación fue levemente mayor que cuando estaban los dos técnicos presentes, pero esta diferencia tampoco fue significativa ( $p=0,3649)$. Las preparaciones de recetas que tenían más de 4 fármacos presentaron un promedio de errores significativamente mayor $(\mathrm{p}=0,0004)$ que aquellas con 3 o menos fármacos (Tabla 7). Sin embargo, el riesgo de errores en la preparación de recetas aumentó 1,8 veces cuando tenía más de tres fármacos (Tabla 8). 
Tabla 7. Distribución y promedio de errores en la preparación de recetas según bloque horario, número de técnicos paramédicos y número de fármacos

\begin{tabular}{|c|c|c|c|c|c|}
\hline Variables & $\begin{array}{l}\text { Frecuencia } \\
\text { de errores }\end{array}$ & $\%$ & $\begin{array}{l}\text { Promedio de } \\
\text { errores }\end{array}$ & $\begin{array}{l}\text { Desviación } \\
\text { Estándar }\end{array}$ & $\mathbf{p}$ \\
\hline \multicolumn{6}{|l|}{ Bloque Horario } \\
\hline Bloque Horario Mañana & 266 & $32,2 \%$ & 0,2 & 0,46 & \multirow{3}{*}{$0,3743^{*}$} \\
\hline Bloque Horario Mediodía & 289 & $34,9 \%$ & 0,3 & 0,53 & \\
\hline Bloque Horario Tarde & 272 & $32,9 \%$ & 0,3 & 0,48 & \\
\hline \multicolumn{6}{|c|}{ Número Técnicos Paramédicos } \\
\hline 1 & 141 & $17,0 \%$ & 0,3 & 0,56 & \multirow[t]{2}{*}{$0,3649 * *$} \\
\hline 2 & 686 & $83,0 \%$ & 0,3 & 0,47 & \\
\hline \multicolumn{6}{|l|}{ Número de fármacos } \\
\hline 1 a 3 & 546 & $66,0 \%$ & 0,2 & 0,43 & \multirow[t]{2}{*}{$0,0004^{* *}$} \\
\hline 4 y más & 281 & $34,0 \%$ & 0,4 & 0,57 & \\
\hline
\end{tabular}

*Kruskal-Wallis. ${ }^{* *}$ Ttest para varianza distintas.

Tabla 8. Riesgo de error en la preparación de recetas con más de tres fármacos. Regresión logística

\begin{tabular}{|lcccc|}
\hline Variable & OR & EE & p & IC (95\%) \\
\hline Bloque Horario Mañana & 0,88 & 0,18 & 0,53 & $0,58-1,32$ \\
\hline Bloque Horario Mediodía & 1,11 & 0,22 & 0,602 & $0,75-1,63$ \\
\hline Número de técnicos paramédicos $=2$ & 1,29 & 0,29 & 0,246 & $0,84-2,00$ \\
\hline Número de fármaco = 3 & 1,76 & 0,3 & 0,001 & $1,26-2,46$ \\
\hline
\end{tabular}

*Referencia: bloque horario tarde. EE: Error estándar.

\section{Discusión}

Respecto a los errores en recetas médicas, de las recetas evaluadas en este estudio, $72,1 \%$ presentaba al menos un tipo de error; cifra menor a lo reportado por otras investigaciones, que van desde $82 \%$ a $90 \%{ }^{15,16}$. Esto es un porcentaje alto considerando que cada error puede suscitar una comprensión errónea de quienes tienen la responsabilidad de interpretar las recetas y despachar los medicamentos incluidos en ellas.

En nuestra investigación, el error más frecuente fue relacionado con la concentración del medicamento prescrito, a diferencia de lo mencionado en la literatura, donde los principales errores en las recetas médicas eran los relacionados a la forma farmacéutica y dosis de los medicamentos. En nuestro estudio, estos errores alcanzaron $15,4 \%$ y $12,5 \%$, respectivamente, cifras diferentes presentadas por otros estudios ${ }^{8,16-19}$. Esto puede explicarse principalmente porque dichos estudios consideraban varios errores en un solo ítem, a diferencia de nuestro estudio que los separaba en varios ítems.

El promedio de errores por receta fue de 2,3 cuando esta tenía más de tres medicamentos prescritos, y 1,6 errores cuando el número de medicamentos fue menor a tres. Estos resultados fueron semejantes a investigaciones que sitúan estas cifras entre 1 y 2,6 errores promedio por prescripción ${ }^{15,16,20}$.

En las recetas con más de dos medicamentos prescritos, el riesgo de error aumenta 2,4 veces. Considerando que en este estudio se encontraron recetas con hasta quince prescripciones, preocupa el hecho que pacientes con muchos fármacos recetados, además de la probabilidad de presentar los problemas propios de una polimedicación (efectos adversos, interacciones, baja adherencia al tratamiento), se deban enfrentar a un mayor riesgo de recetas con errores.

Las recetas correspondientes a altas médicas presentaron el mayor promedio de errores, seguido de las provenientes desde los servicios de medicina interna y urgencia. La inclusión de la 
variable "Procedencia receta médica" en el estudio, se hizo considerando que los tiempos con que cuentan los médicos para atender a los pacientes son diferentes de acuerdo al tipo de atención. Mientras que en los policlínicos de especialidades y medicina interna los horarios de atención son programados y preestablecidos, las atenciones de urgencia, o el alta de un paciente, dependerán de la contingencia y el tiempo utilizado (atención del paciente, emisión receta) estará de acuerdo a la resolutividad de la atención.

La incorporación de sistemas de prescripciones electrónicas mejoraría no sólo los errores asociados a legibilidad, también lo haría con aquellos asociados a la omisión de información, porque el mismo sistema obligaría al prescriptor a completar todos los datos. De esta manera, los sistemas de prescripción computarizados pueden potencialmente mejorar la calidad de los procesos de medicación ${ }^{5,12,21}$.

Respecto a los errores en la preparación de recetas, las preparaciones de recetas que presentaron al menos un error fueron $23,9 \%$ de las evaluadas, a diferencia de otros estudios donde esta proporción llega a $80 \%{ }^{22}$. Esto se debe a que principalmente se trata de estudios desarrollados en recetas de pacientes hospitalizados, por lo que no serían resultados comparables, teniendo en cuenta que los errores pueden ser pesquisados en sala por los profesionales que administran los fármacos, además de la farmacia, existiendo así más instancias de control, aumentando la probabilidad de detección de errores. Por otra parte, hay publicaciones donde los resultados son mucho menores a los nuestros ${ }^{24,25}$, y se debe principalmente a que la información recopilada es en base a reportes de los propios pacientes que se dan cuenta del error al momento de su recepción, o cuando este ingresa a urgencia debido a daño asociado a medicamentos. El registro de datos va a depender si es que el reporte es ejecutado, quedando muchas veces esta información subregistrada.

Los horarios de preparación de recetas y el número de técnicos paramédicos no presentaron diferencias significativas en cuanto a los promedios de errores. Por lo que se deduce que estas variables no influyen en el número de errores.

El número de fármacos por receta fue la variable que presentó diferencias estadísticas significativas, donde el promedio de errores fue mayor en aquellas preparaciones de recetas con más de cuatro fármacos y el riesgo de cometer un error aumentaba 1,8 veces cuando la receta tenía más de tres. Considerando que alrededor de un tercio de las preparaciones estudiadas tenían más de cuatro fármacos, exponen al paciente, además de las desventajas de una polimedicación (mientras más medicamentos, mayor probabilidad de problemas relacionados con la medicación) y lidiar con los tiempos de espera en farmacia, que aumentan cuando mayor es la cantidad de fármacos recetados, se debe agregar el aumento del riesgo de errores al momento de ser preparadas sus recetas.

Los errores de medicación se consideran acontecimientos prevenibles, y si bien muchos de ellos no provocan daño (son leves o no alcanzan al paciente), hay un porcentaje que sí podría potencialmente causar un daño tanto a pacientes como familiares, además de los costos asistenciales y económicos que implicaría. Es por ello que se sugieren medidas como mejoras en los sistemas de almacenamiento, incorporación de sistemas de dispensación automatizados, hasta métodos que mejoren la concentración y comunicación del personal de farmacia. Si bien todas ellas contribuirían a la prevención de errores, lo más importante y que debe estar presente es el chequeo de todas las etapas, especialmente al final del proceso, contando con la presencia de un profesional del área farmacéutica ${ }^{4,6,22,23}$.

Agradecimientos: Se agradece a la dirección del Hospital de Nueva Imperial por colaborar y autorizar la realización de esta investigación, dentro de sus dependencias, confiando en el profesional farmacéutico a cargo, en especial al Director durante el periodo de desarrollo del trabajo, don Víctor Jaramillo. Al Centro de excelencia CIGES (Capacitación, investigación y gestión para la salud basada en evidencia) de la Universidad de La Frontera, por la entrega de las competencias necesarias para el desarrollo de este trabajo a través de su cuerpo docente en el marco de la Maestría en Epidemiología Clínica.

\section{Referencias}

1. Kohn L. Why do errors happen? En: Kohn LT, Corrigan JM, Donaldson MS, Editors, Committee on Quality of Health Care in America, To Err is Human: building a safer health system. Washington D.C, United States: National Academy Press 2000: 49-68. 
2. Adubofour K, Keenan C, Daftary A, Mensah-Adubofour J, Dachman W. Strategies to reduce medication errors in ambulatory practice. J Natl Med Assoc 2004; 96 (12): 1558-64.

3. Otero López MJ. El Papel del farmacéutico en la gestión de la seguridad de los medicamentos diez años después de la publicación del informe "Errar es humano". Farmacia hospitalaria 2010; 34: 159-62.

4. Otero M. La gestión de riesgos en la prevención de los errores de medicación. En: Sociedad Española de Farmacia Hospitalaria, Curso de formación continuada en farmacoterapia de la SEFH. Madrid, España: Aran Ediciones; 2007. p 111-51.

5. Aronson J. Medications errors: definitions and classification. Br J Clin Pharmacol 2009; 67 (6): 599-604.

6. Cheung K, Bouvy M, De Smet P. Medications errors: the importance of safe dispensing. Br J Clin Pharmacol 2009; 67 (6): 676-80.

7. Knudsen P, Herborg H, Mortensen A, Knudsen M, Hellebek A. Preventing medication errors in community pharmacy: frequency and seriousness of medication errors. Qual Saf Health Care 2007; 16: 291-6.

8. Pastó-Cardona L, Masuet-Aumatell C, Bara-Oliván B, Castro-Cels C, Clopés-Estela A, Páez-Vives F, et al. Estudio de incidencia de los errores de medicación en los procesos de utilización del medicamento: prescripción, trascripción, validación, preparación, dispensación y administración en el ámbito hospitalario. Farmacia Hospitalaria 2009; 33 (5): 257-68.

9. Berdot S, Sabatier B, Guillaizeau F, Caruba T, Prognon P. Durieux P. Evaluation of drug administration errors in a teaching hospital. BMC Health Serv Res 2012; 12 (60): 1-8.

10. Dos Santos L, Winkler N, Dos Santos M, Martinbiancho J. Description of medication errors detected at a drug information centre in Southern Brazil. Pharmacy Practice $2015 ; 1: 524$.

11. Tully M. Prescribing errors in hospital practice. Br J Clin Pharmacol 2012; 74 (4): 668-75.

12. Keers R. Williams S. Vattakatuchery J. Brown P. Miller J. Prescott L. Ashcroft D. Prevalence, nature and predictors of prescribing errors in mental health hospitals: a prospective multicentre study. British Medical Journal 2014; 4 .

13. Ryan C, Ross S, Davey P, Duncan E, Francis J, Fielding $\mathrm{S}$, Johnston M, et al. Prevalence and Causes of Prescribing Errors: The PR escribing Outcomes for Trainee Doctors Engaged in Clinical Training (PROTECT)
Study. Plos One 2014; 9 (1).

14. Alvarado C, Ossa X, Bustos L. Adaptación y validación de instrumentos para medir errores en las recetas médicas y en el proceso de preparación de medicamentos en farmacia de pacientes ambulatorios. Rev Med Chile 2014; 142: 1547-52.

15. Lee B, Lehmann C, Jackson E, Kost-Byerly S, Rothman S, Kozlowski L, et al. Assessing Controlled Substance Prescribing Errors in a Pediatric Teaching Hospital: An Analysis of the Safety of Analgesic Prescription Practice in the Transition From the Hospital to Home. The Journal of Pain 2009; 10 (2): 160-6.

16. Graciano M, Borges M, Dean B, Moreira A, Anchieta L, Mota J. Concomitant prescribing and dispensing errors at a Brazilian hospital: a descriptive study. Clinics 2011; 66 (10): 1691-7.

17. Inocenti A, Oliveira R, Camargo A, Pereira D, Escobar F, Trevisan F, et al. Prescription errors in Brazilian hospitals: a multi-centre exploratory survey. Cad Saude Publica 2009; 25 (2): 313-20.

18. Font I, Climent C, Poveda JL. Calidad del proceso farmacoterapéutico a través de errores de medicación en un hospital terciario. Farmacia Hospitalaria 2008; 32 (5): 274-9.

19. Díaz-Carrasco M, Pareja A, Yachachi A, Cortés F, Espuny A. Prescription errors in chemotherapy. Farmacia Hospitalaria 2007; 31 (3): 161-4.

20. Hartel M, Staub L, Röder C, Eggli S. High incidence of medication documentation errors in a Swiss university hospital due to the handwritten prescription process. BMC Health Serv Res 2011; 11 (199): 1-6.

21. Chen Y. Neil K. Avery A. Dewey M. Johnson C. Prescribing error and other problems reported by community pharmacists. Ther Clin Risk Manag 2005; 1 (4): 333-42.

22. Azevedo T, Perini E, Borges M, Comini C. Drug-dispensing errors in the hospital pharmacy. Clinics 2007; 62 (3): 243-50.

23. Beso A, Franklin B, Barber N. The frequency and potential causes of dispensing errors in a hospital pharmacy. Pharm World Sci 2005; 27: 182-90.

24. Cina J, Gandhi T, Churchill W, Fanikos J, McCrea M, Mitton P, et al. How Many Hospital Pharmacy Medication Dispensing Errors Go Undetected? Jt Comm J Qual Patient Saf 2006; 32 (2): 73-80.

25. Assuncao L, Valli C, Pimentel A. Errores de despacho de medicamentos en un hospital público pediátrico. Rev Latino-am Enfermagem 2008 16 (5). Disponible en: www.eerp.usp.br/rlae. 